\title{
Propagating Spin Modes in Canonical Quantum Gravity
}

\author{
Roumen Borissov* and Sameer Gupta ${ }^{\dagger}$ \\ Center for Gravitational Physics and Geometry \\ The Pennsylvania State University \\ University Park, PA 16802
}

\begin{abstract}
One of the main results in canonical quantum gravity is the introduction of spin network states as a basis on the space of kinematical states. To arrive at the physical state space of the theory though we need to understand the dynamics of the quantum gravitational states. To this aim we study a model in which we allow for the spins, labeling the edges of spin networks, to change according to simple rules. The gauge invariance of the theory, restricting the possible values for the spins, induces propagating modes of spin changes. We investigate these modes under various assumptions about the parameters of the model.
\end{abstract}

\footnotetext{
*Present address: Bios Group L.P., Santa Fe, NM 87501, roumen.borissov@biosgroup.com †gupta@phys.psu.edu
} 


\section{Introduction}

There are two very important issues in the loop approach to non-perturbative canonical quantum gravity [1, 2, 3, 4, which we have to address in our quest for a complete theory. The first is the problem of evolution of the gravitational quantum states and the second one is the recovery of the classical continuous space from the discrete structures which appear at very short scale. The problem of evolution in canonical quantum gravity is quite non-trivial. The underlying general covariance principle of the theory leads not to a true Hamiltonian, but to a sum of constraints. The physical states of the theory are then the ones which are annihilated by these constraints. Imposing the gauge and the diffeomorphism constraints has led to a Hilbert space whose basis is given by spin networks space. The Hamiltonian constraint, which generates infinitesimal translations in the time direction, has to be imposed in this space of states in order to obtain the physical space of states.

There are different approaches which have been proposed in order to study the action of the Hamiltonian constraint. In the canonical approach, one relies on regularizing the Hamiltonian constraint as a quantum operator and investigating its action on the states of the theory. Various regularization schemes have been suggested [5, 6], the most commonly accepted being the one proposed by Thiemann[7]. However, all of these suffer from various problems (see for example [8, 9]). Another approach which has been suggested is to look at the finite action of the Hamiltonian constraint as opposed to the infinitesimal one[10]. This has led to the study of spin foams.

An alternative approach is to find a consistent set of rules for quantum evolution which, in the classical limit, recover the action of the Hamiltonian constraint 11]. It is this approach that we study in this paper. While the idea is very intriguing, it is highly non-trivial to define an appropriate set of rules which lead to a good classical limit. The problem of finding the appropriate rules for evolution of the kinematical states is now related to the problem of defining the classical limit of the theory. On the other hand the investigation of the classical correspondence is also quite subtle. One of the main results in canonical quantum gravity is that geometric operators such as area and volume have discrete spectra[12]. This means that space has a discrete structure at the Planck scale. Thus, in taking the classical limit, we should also recover the continuum space-time we see around us.

According to a proposal in [11], the problem of recovering the continuum space is similar to some problems of non-equilibrium critical phenomena in statistical physics. The long range behavior characteristic of classical general relativity could arise at the critical values of some parameters of the discrete theory. This is already seen in certain discrete approaches to quantum gravity such as dynamical triangulations. The important aspect of the proposal is that, since there is no external agent to tune the parameters of the universe, discrete space should be a self-organizing critical system. The idea of self-organized criticality was introduced by Bak, Tang and Wiesenfeld in [13] (for reviews see [14, 15]). The self-organized criticality paradigm is that certain systems, consisting of many parts interacting via local rules, self-organize themselves in a critical state. Usually this critical state is characterized by the lack of specific scale of propagation of perturbations. 
In this paper, we study various models whose dynamics is suggested by canonical quantum gravity. There are certain aspects of the action of the Hamiltonian constraint, which are common to all of the proposed regularizations. These aspects guide us in the introduction of rules for graphical evolution of the spin networks. With the evolution rules in hand, we develop a model along the lines of the sandpile models of self-organized criticality. First we briefly introduce the spin network states. In section 3, we discuss the various regularizations of the action of the Hamiltonian constraint and the corresponding graphical rules for evolution. We then introduce our model and discuss our results.

\section{Spin networks and dual simplicial triangulations}

The basic variables in canonical quantum gravity can be taken to be an $\mathrm{SU}(2)$ connection $A_{a}^{i}(x)$ and a densitized triad $\widetilde{E}_{i}^{a}$ which takes values in the dual of $s u(2)$. A convenient basis for the space of gauge invariant states is given by the spin network states, introduced in canonical quantum gravity by Rovelli and Smolin[16] and by Baez[17]. A spin network state $\Gamma \equiv(\gamma, \vec{j}, \vec{I})$ is defined by the following elements:

- A closed graph $\gamma$, embedded in the spatial manifold $\Sigma$, consisting of finite number of oriented edges $e_{1}, e_{2}, \ldots, e_{n}$ incident at vertices $v_{1}, v_{2}, \ldots, v_{m}$. A vertex is called $p$-valent or of valence $p$ if there are $p$ edges incident at that vertex.

- Each edge $e_{i}$ of the graph $\gamma$ is labeled by an irreducible representation $j_{i}$ of $\mathrm{SU}(2)$. Further on, $c_{i}=2 j_{i}$ is referred to as the color of the edge.

- At each vertex $v_{j}$ there is an intertwining operator from the tensor product of the representations carried by the incoming edges to the tensor product of representations labeling the outgoing edges.

When considering only the combinatorial aspects of a spin network state it is very convenient to think of the spin network on which the state is based as the dual 1-skeleton of a simplicial triangulation of the spatial manifold $\Sigma$. In this picture, a spin network is equivalent to a colored triangulation of the spatial manifold. In the rest of this paper, we shall consider a spin network or its dual triangulation as equivalent objects.

\section{Action of the Hamiltonian constraint}

We shall now schematically describe the graphical action of the Hamiltonian constraint of canonical quantum gravity. Essentially the action of the Hamiltonian constraint can be thought of as adding a loop to the original spin network with some extra requirements. First, the added loop should coincide with two of the edges of the original spin network joined at one vertex in the vicinity of that vertex. Second, the added loop can coincide with any other (portions of) edges. Whenever the added loop runs along already existing edge, the recoupling theory of angular momentum determines the way the original spins change. 


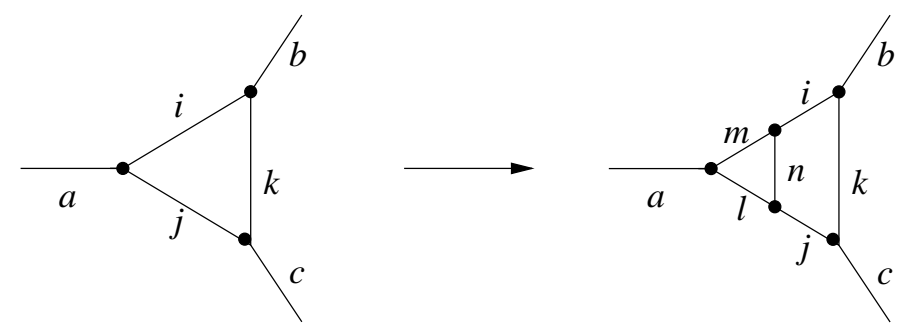

Figure 1: Thiemann's regularization
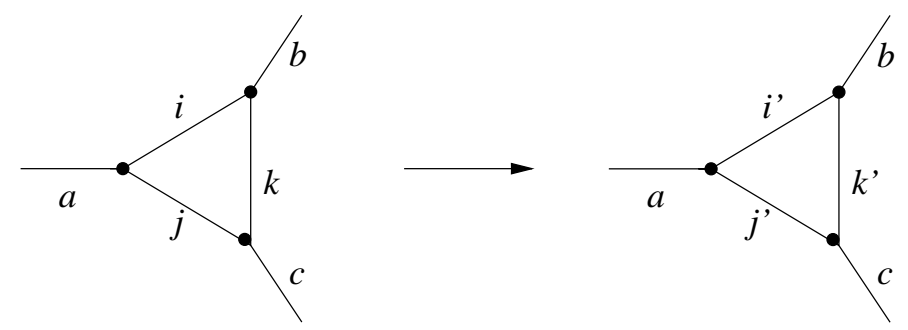

Figure 2: Smolin's regularization

According to the regularization described in [7], the Hamiltonian acts only in a vicinity of the vertices of the spin network. For simplicity let us consider a trivalent vertex. At such a vertex the action of the Hamiltonian constraint can be thought of as a sum of three terms, each one corresponding to the old spin network with an added new edge of color one connecting two of the original edges (see Figure 1). The colors of the original edges in their portion between the newly created vertices and the original vertex are either increased or decreased by one. Consecutive action of the Hamiltonian constraint keeps adding new edges only to the original ones, thus producing a web-like structure at each vertex. The problem with such a definition of the action of the Hamiltonian constraint is that it is ultra-local in the sense that the action of the constraint at one vertex does not propagate any information to the neighboring vertices of the spin network. Hence, there are no long range correlations. A modification which was aimed at fixing this problem was introduced in [8]. This new version of the constraint action allows not only the addition of new edges but also the addition of whole loops coinciding completely with the already present edges. In such cases the original graph does not change at all but the edges of coincidence have their colors changed. This is shown in Figure 2.

In our work, we model the action of the Hamiltonian constraint by introducing a generalization of this second type of regularization. We assume that loops can be added not only to a single closed cycle of edges, but also can run along any collection of the original edges. Also, a very important assumption is that the added loop can run many times along one and the same edge. Since the effect of adding a loop segment to an existing edge simply changes the color of that edge, we can move away from the loop picture and consider simply gauge invariant changes of the colors of the edges of the original spin network. 


\section{The Model}

For simplicity, we study only planar trivalent spin network states in a 2-dimensional space with boundary (topology of a disk). It is convenient to take this space to be a triangle. The spin networks associated with that triangle are based on the graph, dual to the triangulation of the triangle. The triangulation is performed by subdividing the sides of the triangle into $N$ parts. The triangulated triangle is shown in Figure 3. The dual graph on which the spin network states are based is obtained by assigning a vertex to each 2-cell in the triangulation and an edge crossing each 1-cell and connecting the vertices in the neighboring 2-cells (shown using dashed lines in Figure 3). The colors carried by the edges of the spin network can be thought of as being lengths of the corresponding crossed 1-cells of the triangulation [18]. More precisely, the length of a side of the triangulation which is intersected by a spin network edge of color $c$ is

$$
l=\frac{c}{2} l_{\mathrm{P}}
$$

where $l_{\mathrm{P}}$ is the Planck length円. Thus, we shall refer to the triangulation and lengths or its dual spin network and the corresponding colors interchangeably.

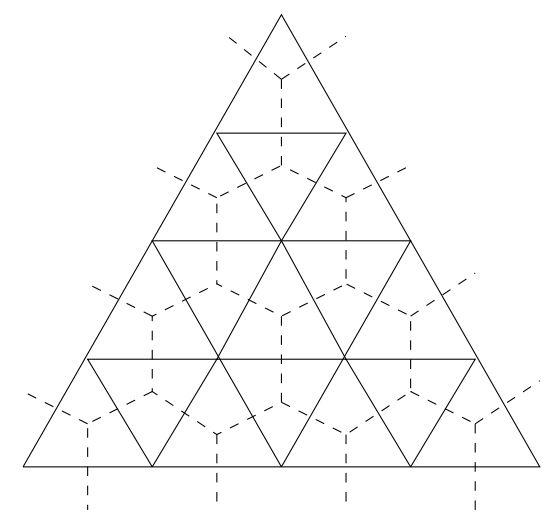

Figure 3: The triangulated triangle and its dual graph

The requirement for gauge invariance at the vertices of the spin network state is essentially a triangle inequality that the lengths of the sides of a triangle should satisfy together with the requirement that the sum of these lengths be an even number. We call the triangle inequality together with the requirement for an even sum of the site's lengths a gauge invariance condition. If $a, b$ and $c$ are the colors of the three edges at a node, the gauge invariance requires that:

$$
\begin{aligned}
a+b & \geq c \\
b+c & \geq a \\
c+a & \geq b \\
a+b+c & =\text { even }
\end{aligned}
$$

\footnotetext{
${ }^{1}$ Recall that in $2 \mathrm{D}, l_{\mathrm{P}}=\hbar G / c^{3}$.
} 
Further, we will assume that the colors of the edges of the spin network crossing the boundary are unconstrained by the evolution process. As an initial state for our system, we construct a spin network based on the dual graph and assign random colors to the edges of the spin network in a gauge invariant fashion. The evolution of spin networks in our model corresponds to changing a set of colors such that the gauge invariance condition remains satisfied at each node of the spin network. We use the following procedure to implement this evolution. Choose, at random, one of the edges of the spin network and change its color by an amount $\Delta c$. This will, in general, affect the gauge invariance of the two nodes on which the edge is incident. To restore the gauge invariance at the nodes, which are no longer gauge invariant, we change the color of one of the remaining incident edges. We continue this process until gauge invariance is restored at every node of the graph.

Depending upon the value of $\Delta c$, there are various possibilities for the exact implementation of the described procedure. For an odd $\Delta c$, say $\Delta c= \pm 1$, the gauge invariance condition is always violated at the two nodes on which the initial edge is incident, because of (3). The restoration of the gauge invariance of the first pair of nodes will necessary induce violation of the gauge condition at two new nodes. There are two cases for this pattern to end - either at a certain step a non-invariant node contains an edge which crosses the boundary of the triangle or the spin changes meet somewhere on the spin network and neutralize each other. These two cases can be viewed from a loop perspective as corresponding to - (i) adding an open line of color 1, whose both ends lie on the boundary or (ii) adding a closed loop of color 1.

The case of even $\Delta c$, say $\Delta c= \pm 2$, is more involved. It is possible that the change of the color of a particular edge by \pm 2 leaves both the original nodes gauge invariant. Actually this is true in most of the cases - requirement (3) is not violated and it is up to the conditions (2) to determine the gauge invariance. We will mostly be interested in this latter case.

Let us now discuss the case $\Delta c= \pm 2$ in more detail. Let us choose an edge of the graph at random and change the length of this edge by \pm 2 . The only case in which this can violate gauge invariance is if the triangle was "flat" initially, that is, if one of the three inequalities in equation (2) is saturated. We call such triangles (vertices) critical. If, for such a critical triangle, we increase the longest side or decrease one of the two shorter sides,
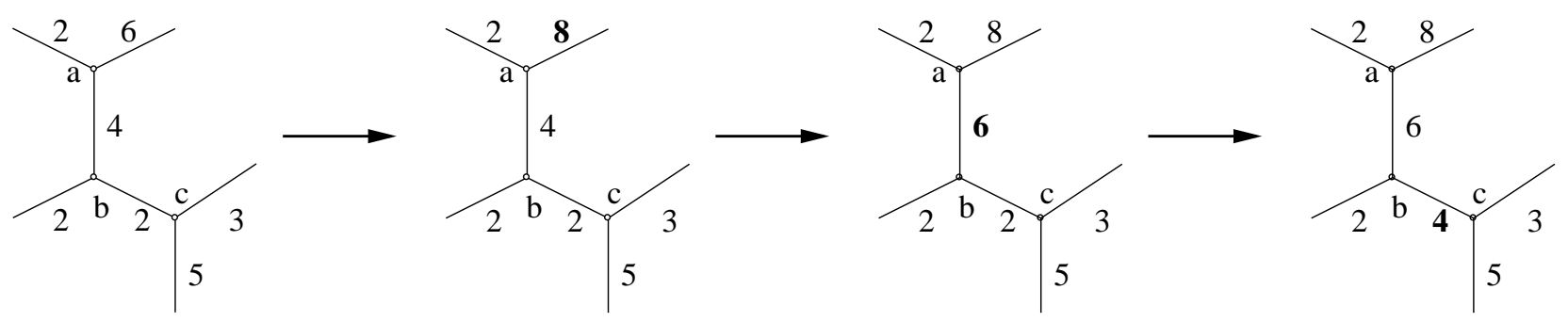

Figure 4: An example of an avalanche of spin changes. The initial perturbation changes the color of the color 6 edge incident at node 'a' to 8 . This makes the node gauge non-invariant and forces the color 4 edge to change to color 6 . This in turn affects node 'b' and so on. 
we will violate gauge invariance. To compensate for this, we have to adjust the length of one of the other two sides. The adjusted side also belongs to one of triangle's neighbors. If this change violates the gauge invariance condition in the neighboring triangle, we adjust the colors there as well. This process will continue until all the triangles in the triangulation satisfy gauge invariance again. Thus, one change of \pm 2 can lead to a series of spin changes (see Figure 1 for an example). These spin changes are analogous to the topplings in the sandpile models. Drawing further analogy with the sandpile models, we refer to the series of spin changes required to restore gauge invariance after the initial change as an avalanche.

Since we allow arbitrary spins to start with, the number of critical triangles will be negligible in comparison to the total number of triangles. Thus if we change the spins in an arbitrary way almost no avalanches will occur. In the sandpile model the analogous situation is when we add and remove grains of sand with equal probabilities. Hence, we introduce a probability $p$ for "flattening" of a triangle. If the arbitrary chosen side has the biggest length in the triangle, we increase the length by 2 with a probability $p$ and decrease it with a probability $1-p$. Similarly if the arbitrary chose side is the smallest one or the one in the middle, we decrease the length by 2 with probability $p$ and increase it with probability $1-p$. Thus it is clear that for $p$ close to 1 , we will be pushing the triangles in the triangulation towards criticality. The rest of the avalanche (if any) proceeds a deterministic way as described above. As a further modification of the above update rule, we introduce the probabilistic update into the entire avalanche. Then, there is a probability $p$ that a gauge non-invariant node is restored to gauge invariance and probability $1-p$ for it to go further away from gauge invariance.

We performed simulations for system sizes ranging from 400 to 10,000 triangles with a typical run covering a million iterations of the system.

\section{Results and Interpretation}

To start with, we simulated the system with the deterministic evolution rule for system size of 400 triangles. The results of these simulations are shown in Figure 5. As can be seen from graph (a) in the figure, the fraction of critical triangles in the system rises very rapidly from an initial value of 0.24 to reach an equilibrium value of around $0.67 \pm 0.01$. The average color per edge in the system increases linearly as the simulation proceeds.

The distribution of avalanches $f(s)$ as function of avalanche size, $s$ is shown in graph (b) of Figure 5. This distribution can be described by an exponential decay

$$
f(s) \sim \exp (-s / \sigma)
$$

to a good accuracy.

The results for a larger system size (10,000 triangles) are shown in Figure 6. The fraction of critical triangles reaches its equilibrium value more slowly as compared to the smaller system. The equilibrium value of $0.70 \pm 0.005$ is similar to that for 400 triangles. As expected, the average color per edge increases at a slower rate as well. The avalanche distribution can 

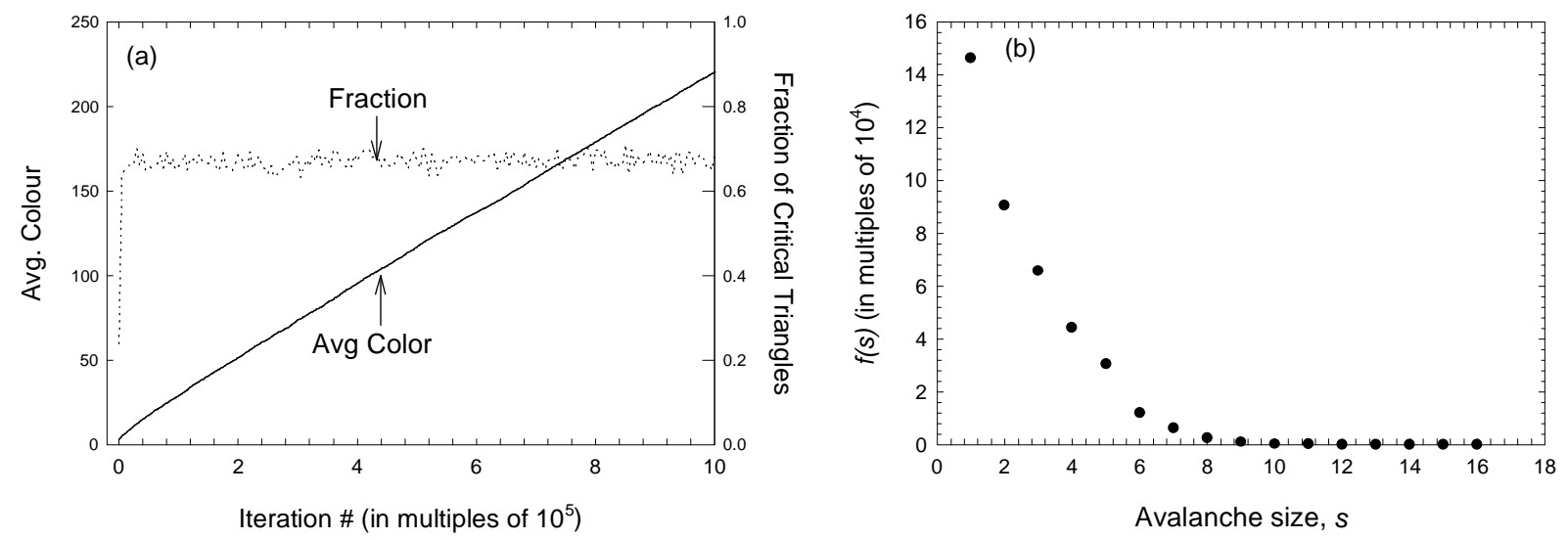

Figure 5: Results for a typical run with 400 triangles. Graph (a) shows how the average color per edge and the fraction of critical triangles in the system evolve as the simulation proceeds. Graph (b) shows the distribution of avalanches $f(s)$ as a function of size.

again be described well by a decaying exponential. Thus, our results scale with the system size.

The simulations for the case where we introduce the probability $p$ into the first move were performed next. Depending upon the value of $p$, a certain fraction of the triangles in the system become critical. Once reached, the fraction of critical triangles remained almost constant. The avalanche distribution was again described by an exponential decay with the decay constant decreasing with decreasing probability. The data remained qualitatively similar to Figures 5 and 6 .

Finally, we simulated the case where the probabilistic update was also included in the avalanche phase of the dynamics. The frequency distribution of the avalanches was still described by an exponential decay. The decay constant however did not decrease as we decreased the probability from 1 . It initially increases till $p=0.4$ and then decreases again (see Figure 7).

Figure 7 seems to indicate that our system undergoes a phase transition around $p=0.4$. However, if we look at the avalanche distribution more carefully, we find that while we get some extremely large avalanches, the number of sites involved in these avalanches is very small. For example, the largest avalanche that we got for the 400 triangles case consisted of 819 topplings which affected only 12 edges. This implies that while we seem to have a phase transition, it does not lead to long range correlations.

\section{Conclusions}

We developed a model of evolving spin networks in which we keep the underlying graph fixed and consider changes of the colors of the edges. In the case when the colors are changed by $\Delta c= \pm 1$, these changes involve either sets of edges which form closed cycles or run between 

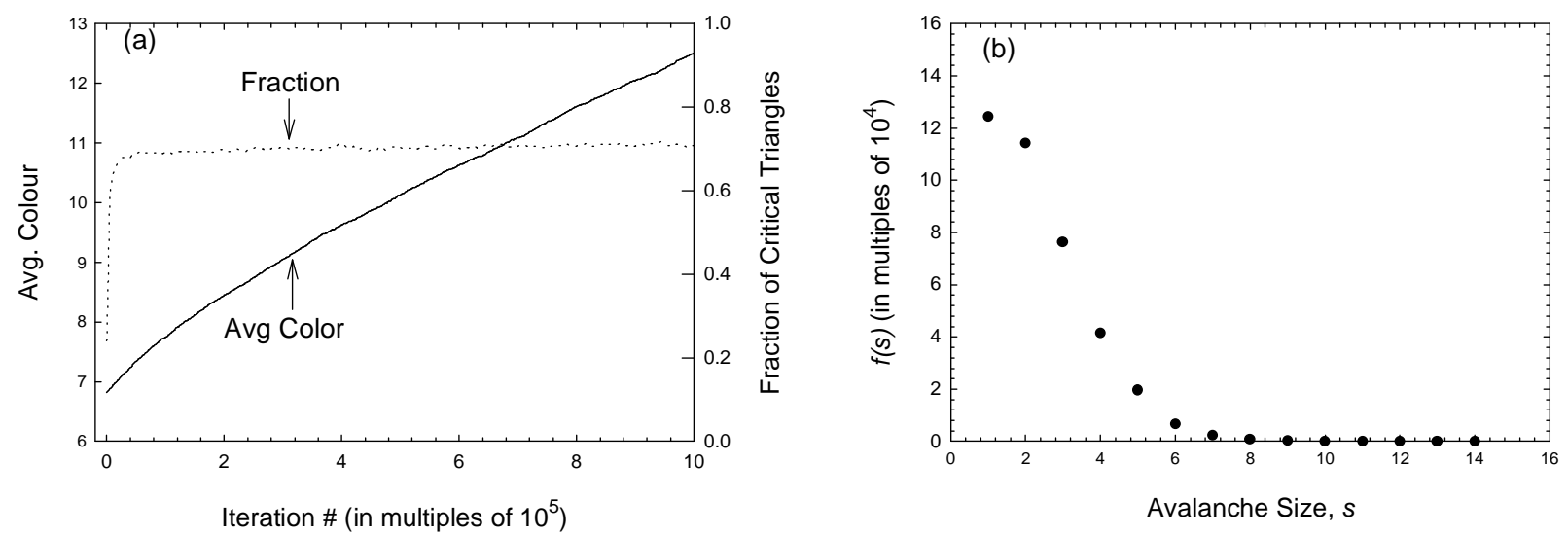

Figure 6: Results for a typical run with 10,000 triangles. Graph (a) shows how the average color per edge and the fraction of critical triangles in the system evolve as the simulation proceeds. Graph (b) shows the distribution of avalanches $f(s)$ as a function of size.

the boundaries of the space. When the colors are changed by $\Delta c= \pm 2$, the response of the system is more involved. Our aim was to prescribe a set of rules for the spin changes and then check if such spin changes would propagate in a scale free behavior, characteristic for a SOC system. The answer to this question to a very large extent depends on the specific features of the model we proposed. It turned out that with the rules we proposed, the system does not exhibit SOC behavior. Instead we found that for particular values of the parameters in the system we got a phase transition. At this point, it cannot be decided using a different set of rules will lead to the kind of self-organized behaviour that we were seeking. There are various possibilities for modification of the evolution rules which we are exploring currently. These include:

- Mixed color updates — for each vertex, at which the gauge condition is violated by

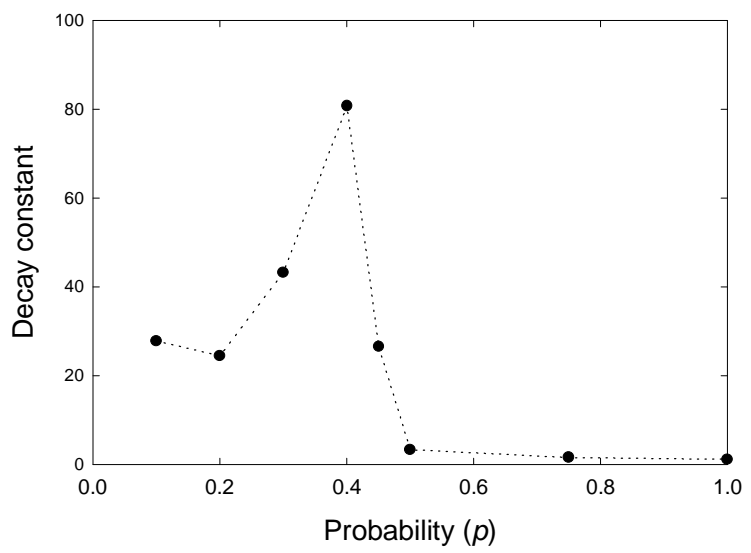

Figure 7: Behaviour of the decay constant as a function of probability 
$\Delta c= \pm 2$ introduce with certain probabilities gauge invariance restoration either by update of the color of one of the edges by $\Delta c= \pm 2$ or of two of the edges by $\Delta c= \pm 1$.

- Conserved total color - after adding a color of 2 to a particular edge, we continue by redistributing the added color among the adjacent edges, thus preserving the total color of the spin network.

- One directional propagation — starting from an edge, one of the nodes of which remains gauge invariant after the spin change. This will avoid the problem of order of overlapping present in the case when the spin changes propagate in two directions.

- Spin changes starting at the boundaries and propagating into the system.

Further work is needed to show if an appropriate set of evolution rules can result in the definition of the spin network states as a self-organizing critical system.

\section{Acknowledgements}

We would like to thank Lee Smolin for the helpful discussions, which led us to this paper and for his important comments. We are also grateful to Fotini Markopoulou and Maya Paczuski for the useful comments and suggestions. This work was supported in part by NSF grant PHY-9514240 to the Pennsylvania State University.

\section{References}

[1] A. Ashtekar, Phys. Rev. Lett. 57 (1986) 2244; Phys. Rev. D36, (1987) 1587.

[2] A. Ashtekar, Lectures on non-perturbative canonical quantum gravity, World Scientific Advanced Series in Astrophysics and Cosmology Vol. 6, Singapore, 1992.

[3] C. Rovelli, Class. Quant. Grav. 8 (1991) 1613.

[4] L. Smolin, in Quantum Gravity and Cosmology, eds. J Pérez-Mercader et al, World Scientific, Singapore 1992.

[5] C. Rovelli and L. Smolin, Phys. Rev. Lett. 72 (1994) 446-449.

[6] R. Borissov, Phys. Rev. D55 (1997) 6099-6111.

[7] T. Thiemann, Class. Quant. Grav. 15 (1998) 839; Class. Quant. Grav. 15 (1998) 875.

[8] L. Smolin, Available as gr-qc/9609034.

[9] R. Gambini, J. Lewandowski, D. Marolf, J. Pullin, Int. J. Mod. Phys. D7 (1998) 97-109.

[10] M. Reisenberger and C. Rovelli, Phys. Rev. D56 (1997) 3490. 
[11] F. Markopoulou and L. Smolin, Nucl. Phys. B508 (1997) 409.

[12] C. Rovelli and L. Smolin, Nucl. Phys. B442 (1995) 593-619.

[13] P. Bak, C. Tang and K. Wiesenfeld, Phys. Rev. Lett. 59 (1987) 381.

[14] P. Bak, How Nature Works, Springer-Verlag, New York 1996.

[15] H. J. Jensen, Self Organized Criticality, Cambridge University Press 1998.

[16] C. Rovelli and L. Smolin, Phys. Rev. D52 (1995) 5743-5759.

[17] J. Baez, Adv. Math. 117 (1996) 253-272 (gr-qc/941107); in "The Interface of Knots and Physics", ed. L. Kauffman, A.M.S., Providence, (1996) 167-203.

[18] C. Rovelli, Phys. Rev. D48 (1993) 2702. 